\title{
The predictive accuracy of sentinel nodes mapping in the setting of pulmonary metastasectomy
}

\author{
Hyun Koo Kim ${ }^{1} \cdot K_{w a n g h y o u n g ~ L e e}{ }^{2} \cdot K_{0 o k}$ Nam Han ${ }^{1} \cdot J_{a e}$ Seon $\mathrm{Eo}^{3} \cdot$ \\ Sungeun $\mathrm{Kim}^{4} \cdot$ Young Ho Choi ${ }^{1}$
}

Received: 28 June 2016 / Accepted: 28 December 2016 / Published online: 6 January 2017

(C) The Author(s) 2017. This article is published with open access at Springerlink.com

\begin{abstract}
This is the first study to evaluate the feasibility of mediastinal lymph node dissection (MLND) based on sentinel lymph node (SLN) status during pulmonary metastasectomy. A total of 22 patients (16 men, 6 women; age $63.3 \pm 7.01$ years) who were candidates for metastasectomy through segmentectomy or lobectomy with MLND owing to cancers metastatic to the lung were enrolled in this study. Radiotracer was administered at the peritumoral region before surgery or soon after initiating surgery. During the operation, the radioactivity of the lymph nodes (ex vivo) was counted with a handheld gamma probe after MLND. Lobectomy was performed in 17 patients, and segmentectomy, in 5 patients. The number of dissected lymph nodes per patient was $14.4 \pm 8.69$ (range, 5-36). In all patients, the SLN could be detected, and the number of SLNs identified was $2.0 \pm 1.15$ (range, 1-5) per patient. Lymph node
\end{abstract}

H. K. Kim and K. H. Lee have contributed equally to this work.

Hyun Koo Kim

kimhyunkoo@korea.ac.kr

Kook Nam Han

hdoccomta@naver.com

1 Departments of Thoracic and Cardiovascular Surgery, Korea University Guro Hospital, Korea University College of Medicine, 97 Guro-donggil, Guro-gu, 152-703 Seoul, South Korea

2 Departments of Thoracic and Cardiovascular Surgery, Korea University Anam Hospital, Korea University College of Medicine, Seoul, South Korea

3 Departments of Nuclear Medicine, Korea University Guro Hospital, Korea University College of Medicine, Seoul, South Korea

4 Departments of Nuclear Medicine, Korea University Anam Hospital, Korea University College of Medicine, Seoul, South Korea metastasis was identified in 3 of the 22 patients (13.6\%), and none of the 3 patients with N1 or N2 disease had falsenegative SLNs. SLN identification might be an indicator of whether or not MLND should be performed during pulmonary metastasectomy. However, further large-volume and multi-institutional studies are needed.

Keywords Cancers metastatic to the lung .

Metastasectomy $\cdot$ Mediastinal lymph node dissection

\section{Introduction}

The lung is one of the most common sites of distant metastasis of solid tumors because the pulmonary arterial vascular bed is the first filter in the hematogenous route [1]. More than $30 \%$ of extra-thoracic malignances give rise to pulmonary metastasis during the course of the disease, and approximately $20 \%$ of these cases feature metastases that are confined to the lungs [2].

Pulmonary metastasis represents terminal systemic disease; hence, chemotherapy has been the mainstay of therapeutic intervention in the majority of cases [3]. However, surgical resection of pulmonary metastasis is sometimes necessary for pathological confirmation, and complete surgical excision of all pulmonary deposits is often technically feasible with low morbidity and mortality. Improvement of surgical techniques such as parenchymal-sparing resection, development of more effective chemotherapy regimens, and significantly increased long-term survival as observed in retrospective studies have resulted in pulmonary metastasectomy gradually becoming a standard therapeutic procedure in properly selected patients $[1,4]$. The theoretical basis of local treatment, such as surgery with a curative intent, for metastatic tumor is the "cascade-spreading 
process" of cancer cells to other organs. Even with hematogenous metastasis, there may be a presystemic stage, that is, tumor spread limited to "key-site" organs such as the liver or lung $[5,6]$.

The incidence of thoracic lymph node involvement in pulmonary metastasis ranges from 5 to $66.3 \%$, and the presence of lymph node metastasis is a significant factor for worse prognosis [7, 8]. The addition of mediastinal lymph node dissection (MLND) to pulmonary metastasectomy remains controversial, because its therapeutic efficacy is insufficient and it is associated with a longer operation time [9]. However, local recurrence seems to decrease when MLND is performed during pulmonary metastasectomy [10, 11]. Additionally, performing lymph node dissection or sampling can reveal unexpected lymph node involvement and provide useful information for postoperative therapeutic decisions [7, 12].

The sentinel lymph node (SLN) is the first pass of lymphatic drainage for cancer cells and is the key lymph node for acquiring information regarding the presence of metastasis from the primary tumor. If metastasis in the SLN is not identified, it is possible to avoid unnecessary lymph node dissection [13]. SLN biopsy has become a standard procedure in the treatment of early stage breast cancer and melanoma as a way to avoid the complications of lymph node dissection. Although the application of SLN mapping to lung cancer surgery has remained controversial, it could be helpful in deciding whether omission of MLND is possible or whether segmentectomy is indicated in patients with clinically node negative disease [14].

The hypothesis of this study was that regional lymph node metastasis in patients with cancers metastatic to the lung occurs in a manner similar to lymph node metastasis in primary lung cancer; hence, SLN identification might be an indicator of whether MLND should be performed or not during pulmonary metastasectomy. However, it is unclear whether SLN identification has the same significance in the setting of hematogenous spread of an extrathoracic primary cancer. This is the first study to evaluate the feasibility of MLND based on SLNs during pulmonary metastasectomy in patients with clinically node negative cancers metastatic to the lung.

\section{Materials and methods}

Candidates for this study included patients who underwent pulmonary metastasectomy with MLND with a curative intent for cancers metastatic to the lung between March 2008 and November 2013 at the Korea University Guro Hospital. After approval by the Ethics Committee of the institution, written informed consent was obtained from all patients in accordance with the Declaration of Helsinki (KUGH14230-001).

For preoperative assessment, each patient underwent thoracic computed tomography (CT) scanning, positron emission tomography (PET/CT), and a pulmonary function test. In principle, the diagnosis and treatment plan for pulmonary metastasis were confirmed by a multidisciplinary tumor board including thoracic surgeons, radiologists, nuclear medicine specialists, and medical oncologists. Pulmonary metastasis was diagnosed on the basis of tissue confirmation through percutaneous needle biopsy under CT-guidance, or on the basis of radiologic confirmation through revaluation after an observation period of at least 2 months after initial diagnosis.

The inclusion criteria in this study followed the basic principles for selecting patients to undergo pulmonary metastasectomy, which were technical resectability, tolerable general and functional surgical risk, control of the primary tumor, and no further extrathoracic metastasis [15]. Patients who had received chemotherapy before pulmonary metastasectomy as neoadjuvant chemotherapy for pulmonary metastasis or repeat pulmonary metastasectomy were not included. In addition, patients with suspicion of metastasis in the hilar or mediastinal lymph nodes as observed on CT or PET/CT were excluded from this study. Mediastinoscopic or endobronchial ultrasonic mediastinal LN biopsy was not performed in any patient. Patients with a single pulmonary metastasis or multiple metastases in the same lobe were included, but patients with multiple metastases in different lobes were excluded.

In our hospital, when a metastasis is located at the peripheral lung and there is no evidence of other pulmonary metastasis, in most cases, we attempt to apply a minimally invasive surgical approach such as video-assisted thoracoscopic (VATS) wedge resection after preoperative localization under CT guidance [16, 17]. MLND is not performed in these patients in order to minimize complications after surgery. However, when the metastasis is located in the central lung, and the resection margin is supposed to be more than $2 \mathrm{~cm}$, segmentectomy is performed. When the resection margin is not sufficient in centrally located metastases, or there is a large tumor or multiple metastases in the same lobe, lobectomy is chosen. VATS major pulmonary resection is performed, regardless of etiology, when the lesion is amenable to anatomic resection and the patient can tolerate single lung ventilation, as determined by preoperative pulmonary function tests in our institution [18, 19]. VATS was not performed in cases of a definite pleural calcification, tight calcification adherent to a pulmonary vessel, thoracic cage deformity, decreased ipsilateral lung volume, a tumor $6 \mathrm{~cm}$ or larger, an invasive mediastinal tumor, or the inability to completely resect the lesion. Eventually, patients who underwent segmentectomy or lobectomy as 
well as complete MLND by VATS or standard thoracotomy were included in this study.

The methods of radioisotope injection and intraoperative SLN mapping were the same as in previous studies [20, 21]. A total dose of $1 \mathrm{mCi}$ of radiotracer $\left({ }^{99 \mathrm{~m}} \mathrm{Tc}\right.$-mannosyl human serum albumin; MSA or ${ }^{99 \mathrm{~m}}$ Tc-phytate) in $0.2 \mathrm{ml}$ was administered at the peritumoral region under chest $\mathrm{CT}$ guidance approximately $1 \mathrm{~h}$ before surgery in the CT room, or soon after placing ports for video-assisted thoracoscopic surgery or standard thoracotomy during surgery according to the availability of the CT room on the day of surgery. When patients had multiple metastases in the same lobe, radiotracer was injected at the largest lesion. Radioactivity in the lymph nodes was counted after dissection with a handheld gamma probe (Neo2000; Johnson \& Johnson, Cincinnati, OH). SLN was defined as any node for which the count was five times the radioactivity of the resected lung tissue with the lowest count. All collected lymph nodes including SLNs were cut into $2 \mathrm{~mm}$ slices and diagnosed from formalin-fixed and paraffin-embedded sections stained with hematoxylin and eosin.

\section{Statistical analysis}

The identification rate was defined as the percentage of patients with detected SLNs among the entire analyzed group. The false-negative rate for SLN identification was assessed by the presence of metastatic lymph nodes not identified as SLNs, with the labeled SLNs histologically appearing uninvolved. A $p$ value of $<0.05$ was considered statistically significant. Statistical software (SPSS for Windows, version 22.0; SPSS, Chicago, IL) was used for statistical analysis.

\section{Results}

A total of 22 patients ( 16 men, 6 women) were enrolled in this study, and their mean age was $63.3 \pm 7.01$ years (range 52-76). The primary lesions of cancers metastatic to the lung involved the rectum (adenocarcinoma) in 8 patients, the colon (adenocarcinoma) in 8 patients, the kidney (renal cell carcinoma) in 2 patients, the skin (malignant granular cell tumor) in 1 patient, the thigh (sarcoma) in 1 patient, the thyroid (papillary carcinoma) in 1 patient, and the endometrium (adenocarcinoma) in 1 patient (Table 1). The pulmonary metastases were located in the right upper lobe in 4 patients, right middle lobe in 4 patients, right lower lobe in 9 patients, left upper lobe in 3 patients, and left lower lobe in 2 patients. Lobectomy was performed in 17 patients, and segmentectomy, in 5 patients. All patients had a single pulmonary metastasis, except 2 patients who had multiple metastases in the
Table 1 Patient characteristics

\begin{tabular}{ll}
\hline Characteristic & No. \\
\hline Sex (Male/Female) & $16 / 6$ \\
Age, years & $63.3 \pm 7.01$ (52-76) \\
Primary lesion (pathology) & \\
Rectum (adenocarcinoma) & 8 \\
Colon (adenocarcinoma) & 8 \\
Kidney (renal cell carcinoma) & 2 \\
Skin (malignant granular cell tumor) & 1 \\
Thigh (sarcoma) & 1 \\
Thyroid (papillary carcinoma) & 1 \\
Endometrium (adenocarcinoma) & 1 \\
Tumor location & \\
Right upper & 4 \\
Right middle & 4 \\
Right lower & 9 \\
Left upper & 3 \\
Left lower & 2 \\
Tumor size, cm & $2.7 \pm 1.90$ (0.9-7.5) \\
Approach & \\
VATS & 17 \\
Thoracotomy & 5 \\
Surgery & \\
Lobectomy & \\
Segmentectomy & \\
\hline
\end{tabular}

Data are presented as $\mathrm{n}$ or mean \pm standard deviation (range)

VATS video-assisted thoracoscopic surgery

same lobe and underwent lobectomy. The size of the pulmonary metastasis was $2.7 \pm 1.90 \mathrm{~cm}(0.9-7.5)$.

The number of dissected lymph nodes per patient was $14.4 \pm 8.69$ (range 5-36) (Table 2). In all patients, the SLN could be detected (SLN identification rate, 100\%), and the number of SLNs identified was $2.0 \pm 1.15$ (range $1-5)$ per patient. SLNs were detected at the mediastinal lymph node station in 17 out of 22 patients (77.3\%), and at the hilar lymph node station in 14 out of 22 patients (63.6\%). Eight out of 22 patients (36.4\%) had SLNs only in the mediastinum, 5 patients $(22.7 \%)$ had SLNs only in the hilar lymph node station, and 9 patients (40.9\%) had SLNs in both the mediastinal and hilar lymph node stations. The distribution of mediastinal SLNs is shown in Fig. 1. In 19 out of 22 patients (86.4\%), mediastinal SLNs were located in a lobe specific area. In 3 patients $(13.6 \%)$, mediastinal SLNs were located in the non-lobe specific area; one patient with cancer metastatic to the lung, involving the right upper lobe, had a SLN at the inferior pulmonary ligament, one patient with metastasis in the right upper lobe had a SLN at the subcarinal area, and one patient with metastasis in the left upper lobe had a SLN in the subcarinal area. 
Table 2 Results of SLN identification

\begin{tabular}{ll}
\hline Characteristic & No. \\
\hline Dissected LN (No.) & $14.4 \pm 8.69(5-36)$ \\
SLN detection rate (\%) & $100(22 / 22)$ \\
No. of SLNs & $2.0 \pm 1.15(1-5)$ \\
Metastasis (no. of patients) & $3 / 22(13.6 \%)$ \\
False-negative SLNs (\%) & 0 \\
\hline
\end{tabular}

Data are presented as $\mathrm{n}$ or mean \pm standard deviation (range)

SLN sentinel lymph node

Lymph node metastasis was identified in 3 of the 22 patients (13.6\%), and skipped metastasis was not noted in any case (Table 3 ). Among the 3 patients, lymph node metastases were found at lobe-specific areas in 2 patients, but one patient with clinical stage T3N0M0 disease had metastasis in a non-lobe specific mediastinal lymph node as well as in a lobe specific mediastinal lymph node. However, none of the 3 patients with $\mathrm{N} 1$ or N2 disease had false-negative SLNs.

No complications were observed during the SLN mapping procedure. There was no mortality or major perioperative morbidity, only minor complications such as prolonged air leak in 2 patients $(0.9 \%)$ and pneumonia in $1(0.5 \%)$.

During the follow-up period $(41.5 \pm 26.1$ months $)$, the 3-years thoracic recurrence-free rate was $43.5 \%$ in all patients, and there was a potential difference between pathologically lymph node positive and negative cases (54.4\% in node negative cases vs. $33.3 \%$ in node positive, $p=0.07$ ) (Fig. 2). The 3 -year overall survival rate was $70.6 \%$ in all patients, and there was a potential difference between
Fig. 1 Distribution of sentinel nodes according to tumor location. a Right upper lobe, $\mathbf{b}$ right middle lobe, $\mathbf{c}$ right lower lobe, d left upper lobe, e left lower lobe. In patients with a metastatic lung cancer in the right upper lobe, positive sentinel nodes were mostly detected in the paratracheal and interlobar lymph nodes (10/12, 83.3\%).

Two sentinel nodes (16.7\%) were not located in lobe-specific areas such as the subcarinal and inferior pulmonary ligament area. Metastases were found only in non-lobe specific areas

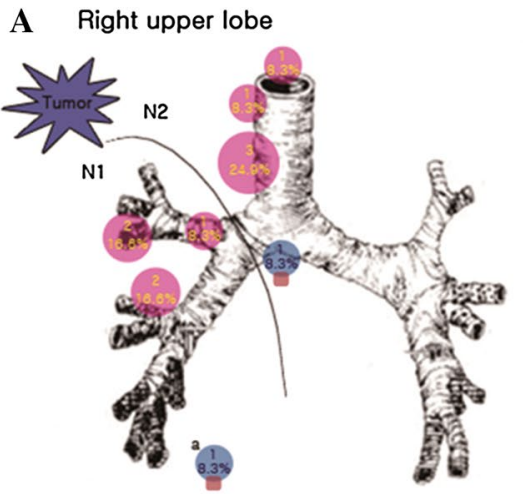

D Left upper lobe

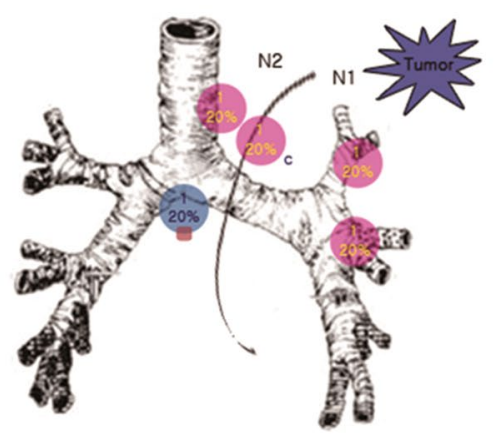

B Right middle lobe

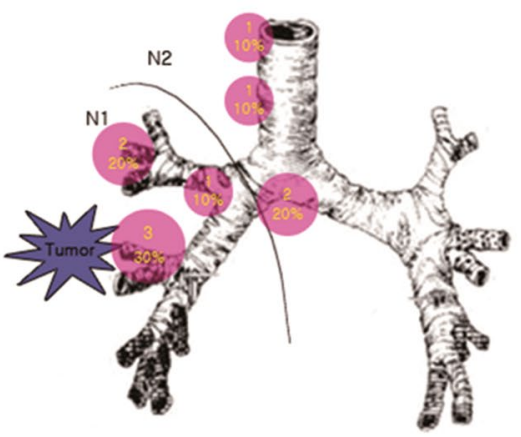

C Right lower lobe

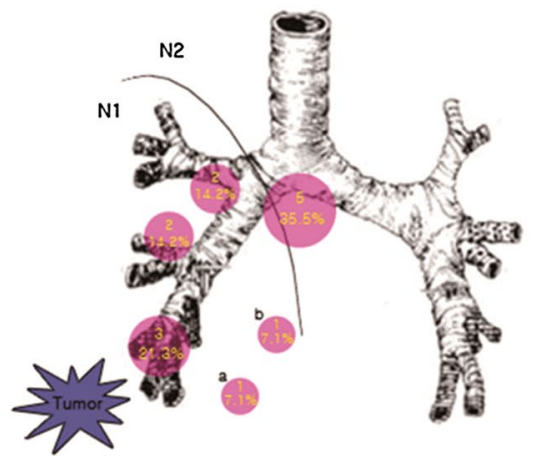

E Left lower lobe

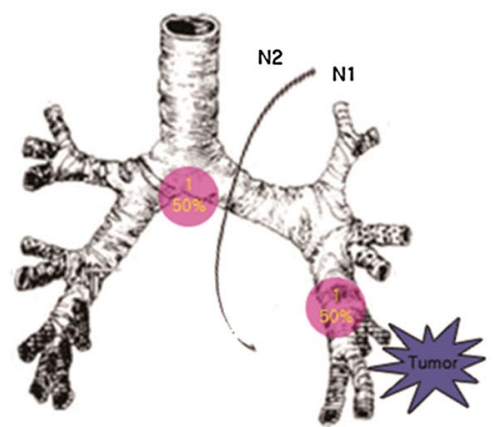

a. Right pulmonary ligament lymph node

b. Right paraesophageal lymph node

c. Subaortic lymph node

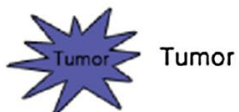

Sentinel lymph node (\%) - lobe specific

Sentinel lymph node (\%) - non lobe specific

Lymph node metastasis 
Table 3 Characteristics of the patients with lymph node metastasis during pulmonary metastasectomy

\begin{tabular}{lllllll}
\hline Sex & Age (years) & Primary cancer & Affected lobe & $\begin{array}{l}\text { Tumor size } \\
(\mathrm{cm})\end{array}$ & SLN station & $\begin{array}{l}\text { Metastatic } \\
\text { LN station }\end{array}$ \\
\hline Female & 60 & Rectum & Right upper & 4 & $9,11,12$ & 11 \\
Male & 73 & Colon & Right lower & 7.5 & 4 & $4,7,10$ \\
Male & 56 & Colon & Right lower & 3.5 & 7 & 7,11 \\
\hline
\end{tabular}

SLN sentinel lymph node
A Thoracic recurrence free

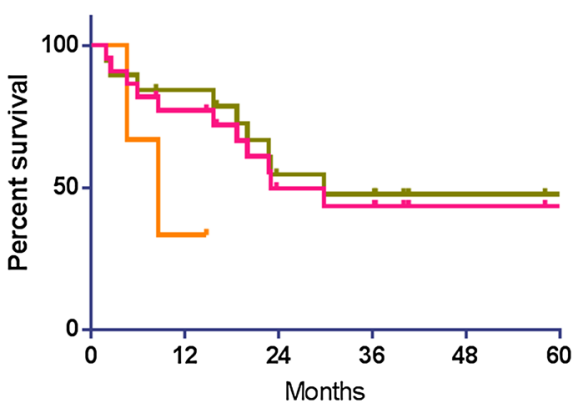

Fig. 2 The 3-year thoracic recurrence-free rate and overall survival rate after pulmonary metastasectomy with mediastinal lymph node dissection in clinically node negative patients. The 3 years thoracic

pathologically lymph node positive and negative cases (77.0\% in node negative cases vs. $33.3 \%$ in node positive, $p=0.05)$.

\section{Discussion}

MLND may contribute to complete carcinologic control and more accurate patient prognosis, which allows for customized adjuvant treatment even in patients with thoracic lymph node involvement during pulmonary metastasectomy [10]. However, the presence of clinical or pathological lymphadenopathy is still believed to be a relative or absolute contraindication to pulmonary metastasectomy by most thoracic surgeons [22], because the presence of lymph node metastasis is a significant and independent poor prognostic factor in patients with pulmonary metastasis $[7,11$, 12]. Moreover, the role of MLND during pulmonary metastasectomy has not been established because its prognostic significance is controversial.

The policy of our hospital is that patients with suspicion of metastasis in the hilar or mediastinal lymph nodes as observed on preoperative CT or PET/CT are not candidates for pulmonary metastasectomy. However, preoperative imaging, such as CT or PET/CT, is limited in determining the presence of metastasis in thoracic lymph nodes, and Seebacher et al., reported that MLND during pulmonary metastasectomy revealed unexpected lymph node
B

\section{Overall survival}



recurrence-free rate and overall survival rate was 43.5 and $70.6 \%$, respectively. There were potential differences between pathologically lymph node positive and negative cases

involvement in $17 \%$ of patients with clinically negative lymph nodes [12]. Kudelin et al. recommend MLND for every patient due to the high prevalence of thoracic lymph node involvement [23]. Given the limitations of these preoperative imaging techniques and the positive impact of subsequent treatment, MLND at the time of metastasectomy appears reasonable in patients suspected to have no thoracic lymph node involvement [12, 13]. However, careful consideration of the need for MLND is required in all patients, because of the increased morbidity and decreased quality of life associated with it. It is thus important for intraoperative decision making regarding MLND to be performed for each patient with pulmonary metastatic disease.

To reduce unnecessary lymph node dissection, many feasibility studies of MLND based on SLN have been conducted for primary lung cancer [24, 25], and we have extensive experience in SLN mapping [14, 20, 26]. We hypothesized that SLN identification might be an indicator of whether or not MLND should be performed during pulmonary metastasectomy if regional lymph node metastasis in cancers metastatic to the lung occurs in a manner similar to lymph node metastasis in primary lung cancer. However, it is unclear whether SLN identification has the same significance in the setting of hematogenous spread of an extrathoracic primary cancer.

In this study, SLN was identified in all cases, and lymph node metastasis was found only in SLNs (no false positives). This result indicates that SLNs identified 
during pulmonary metastasectomy alone might possibly be metastatic; hence, the decision regarding the performance of MLND could be made during surgery. Furthermore, lymph node sampling or dissection could provide more accurate staging and be used to determine the appropriate adjuvant treatment, but the effects of sampling and dissection on long term survival do not significantly differ $[7,10,12]$. Therefore, obtaining only the SLN during pulmonary metastasectomy might be sufficient to gain accurate information regarding disease status and similar long term survival comparing to MLND. Although lymph node metastasis was identified in 3 patients and skipped metastasis was not noted in any patient $(0 \%)$ in this study, Pfannschmidt et al. reported that $16.3 \%(13 / 45)$ of patients had mediastinal noncontiguous lymph node spread [27]. However, a large-scale study is needed to address this issue in the future.

This study has many limitations, including the small number of patients, heterogeneity of the primary cancers, the single center experience, and the fact that patients who underwent wedge resection were not included. These limitations should be taken into consideration when interpreting the results of this study.

In conclusion, SLN identification might be an indicator of whether or not MLND should be performed during pulmonary metastasectomy. However, further large-volume and multi-institutional studies are needed to confirm this finding.

Funding This work was supported by a Grant from the Korean Health Technology R\&D Project, Ministry of Health \& Welfare, Republic of Korea (No. A121074) and a National Research Foundation of Korea (NRF) Grant funded by the Ministry of Education, Science and Technology (No. NRF-2015R1A2A2A04005760).

Author contributions Prof. HKK contributed to the surgical procedure, reviewing data, and drafting the article. Dr. KL contributed to reviewing data and drafting the article. Prof. KNH contributed to reviewing data. Prof. JSE contributed to the procedure and reviewing data. Prof. SK contributed to the procedure and reviewing data. Prof. YHC contributed to the surgical procedure and reviewing data.

\section{Compliance with ethical standards}

Conflict of interest The authors declares that they have no conflict of interest.

Open Access This article is distributed under the terms of the Creative Commons Attribution 4.0 International License (http:// creativecommons.org/licenses/by/4.0/), which permits unrestricted use, distribution, and reproduction in any medium, provided you give appropriate credit to the original author(s) and the source, provide a link to the Creative Commons license, and indicate if changes were made.

\section{References}

1. Kruger M, et al (2014) Optimal timing of pulmonary metastasectomy-is a delayed operation beneficial or counterproductive? Eur J Surg Oncol 40(9):1049-1055

2. Pastorino U (1997) Lung metastasectomy: why, when, how. Crit Rev Oncol Hematol 26(3):137-145

3. Deo SS, et al. (2014) Pulmonary metastasectomy: review of experience at a tertiary cancer care center. J Cancer Res Ther 10(3):535-539

4. Casiraghi $\mathrm{M}$ et al (2011) A 10-year single-center experience on 708 lung metastasectomies: the evidence of the "international registry of lung metastases". J Thorac Oncol 6(8):1373-8

5. Viadana FB, Pickren JW (1978) Cascade spread of bloodborne metastasis in solid and nonsolid cancers of humans. In: Weiss L, Gilbert HA (eds) Pulmonary metastasis. G. K. Hall. Medical Publications Division, Boston, pp 143-157

6. Suemasu K et al (1987) [Clinical and basic studies on the treatment of cancer metastasis]. Gan To Kagaku Ryoho 14(3 Pt 1):561-566

7. Shiono $\mathrm{S}$ et al (2015) The prognostic impact of lymph-node dissection on lobectomy for pulmonary metastasis. Eur J Cardiothorac Surg 48(4):616-621, (discussion 21)

8. Garcia-Yuste M, Cassivi S, Paleru C (2010) Thoracic lymphatic involvement in patients having pulmonary metastasectomy: incidence and the effect on prognosis. J Thorac Oncol 5(6):S166-S169

9. Allen MS et al (2006) Morbidity and mortality of major pulmonary resections in patients with early-stage lung cancer: initial results of the randomized, prospective ACOSOG Z0030 trial. Ann Thorac Surg 81(3):1013-1019, (discussion 9-20)

10. Renaud S et al (2014) Systematic lymph node dissection in lung metastasectomy of renal cell carcinoma: an 18 years of experience. J Surg Oncol 109(8):823-829

11. Hamaji $\mathrm{M}$ et al (2012) Is lymph node dissection required in pulmonary metastasectomy for colorectal adenocarcinoma? Ann Thorac Surg 94(6): 1796-800

12. Seebacher $G$ et al (2015) Unexpected lymph node disease in resections for pulmonary metastases. Ann Thorac Surg 99(1):231-236

13. Shayan R, Achen MG, Stacker SA (2006) Lymphatic vessels in cancer metastasis: bridging the gaps. Carcinogenesis 27(9):1729-38

14. Kim HK et al (2012) Comparison between preoperative versus intraoperative injection of technetium-99 m neomannosyl human serum albumin for sentinel lymph node identification in early stage lung cancer. Ann Surg Oncol 19(4):1343-1349

15. Mountain CF, McMurtrey MJ, Hermes KE (1984) Surgery for pulmonary metastasis: a 20-year experience. Ann Thorac Surg 38(4):323-30

16. Doo KW et al (2015) Needlescopic resection of small and superficial pulmonary nodule after computed tomographic fluoroscopy-guided dual localization with radiotracer and hookwire. Ann Surg Oncol 22(1):331-337

17. Kang DY et al (2011) Needlescopy-assisted resection of pulmonary nodule after dual localisation. Eur Respir J 37(1):13-7

18. Kim HK, Choi YH (2015) The feasibility of single-incision video-assisted thoracoscopic major pulmonary resection performed by surgeons experienced with a two-incision technique. Interact Cardiovasc Thorac Surg 20(3):310-5

19. Kim HK et al (2013) The feasibility of a Two-incision videoassisted thoracoscopic lobectomy. J Cardiothorac Surg 8:88

20. Kim $\mathrm{S}$ et al (2010) Intra-operative sentinel lymph node identification using a novel receptor-binding agent (technetium99m neomannosyl human serum albumin, 99mTc-MSA) in 
stage I non-small cell lung cancer. Eur J Cardiothorac Surg 37(6):1450-1456

21. Kim H et al (2011) A comparative study of two- versus onelung ventilation for needlescopic bleb resection. Eur Respir $\mathbf{J}$ 37(5):1183-1188

22. Internullo E et al (2008) Pulmonary metastasectomy: a survey of current practice amongst members of the European society of Thoracic Surgeons. J Thorac Oncol 3(11):1257-66

23. Kudelin N et al (2013) Metastasectomy with standardized lymph node dissection for metastatic renal cell carcinoma: an 11-year single-center experience. Ann Thorac Surg 96(1):265-270, (discussion 70-1)
24. Nomori $\mathrm{H}$ et al (2011) Sentinel nodes in lung cancer: review of our 10-year experience. Surg Today 41(7):889-895

25. Liptay MJ et al (2002) Intraoperative sentinel lymph node mapping in non-small-cell lung cancer improves detection of micrometastases. J Clin Oncol 20(8):1984-1985

26. Eo JS et al (2015) Gallium-68 neomannosylated human serum albumin-based PET/CT lymphoscintigraphy for sentinel lymph node mapping in non-small cell lung cancer. Ann Surg Oncol 22(2):636-641

27. Pfannschmidt $\mathbf{J}$ et al (2006) Nodal involvement at the time of pulmonary metastasectomy: experiences in 245 patients. Ann Thorac Surg 81(2):448-454 